\title{
Existence and Extremal Solution of Boundary Value Problem for Nonlinear Hybrid Fractional Differential Equation in Banach Algebras
}

\author{
B.D. Karande \& Pravin M. More
}

Department of Mathematics, Maharashtra Udayagiri Mahavidyalaya Udgir, India

Department of Mathematics, B.S.S.ASC College Makni, India

Email:pravinmorepravin@gmail.com

Corresponding Author: Pravin M. More

\begin{abstract}
In this work we study the existence and extremal solution for the boundary value problem of the nonlinear hybrid fractional differential equation by using hybrid fixed point theorem in Banach Algebra due to Dhage's theorem.
\end{abstract}

Keywords: Banach algebra, Hybrid fixed point theorem, Existence result, Locally attractive solution, Extremal solution

\section{Introduction}

The concept of fractional calculus has gained considerable popularity and importance during the past ten decades and has numerous applications in diverse fields of science and engineering. The credit of theory of fractional derivatives goes to L' Hospital (1661-1704) in denotation of the derivative of order

$1 / 2$. The theory of derivative and integral of arbitrary order in subsequent mentioned of factional derivatives was made in same contexts by Euler (1730), Lagrange (1772), Lacriox (1819), Fourier (1822), Laplace (1832), Liouville (1832), Riemann (1847), Greer (1859), Holmgren (1865), Grunwald (1867), Letnikov(1868), Sonin (1869), Laurent (1884), Nekrassov(1888),Krug (1890) and Wely (1917) etc. Recently there are several works on fractional derivatives and integral equations, for instances, we refer a few (see, [1],[8],[9],[13]). The theory of fractional derivative and integral are very suitable for solving linear and nonlinear functional equations.

Bhi and Lu [1] and Zhao and Wang [18] studied the following two point boundary value problem of fractional differential equation

$$
D_{0^{+}}^{\alpha} u(t)+f(t, u(t))=0 \quad 0<t<1, \quad u(0)=u(1)=0
$$

where $1<\alpha \leq 2$ is a real number and $D_{0^{+}}^{\alpha}$ is the standard Riemann-Liouville fractional derivative. By the properties of Green function, they have some multiple positive solutions for singular and nonsingular boundary value problem by mean of Lery-Schauder fixed point theorem on cone. They obtained the existence of positive solutions by using Guo-Krasnosel'kii fixed point theorem and Leggett-Williams fixed point theorem. In the recent years, quadratic perturbations of nonlinear differential equations have attracted much attention to many researchers. We call such differential equations as hybrid differential equations and have been studied in some works, for instances: [2],[11],[12],[14],[15] and [17].

Zhao et al [3] studied first order hybrid differential equations (In shorts FHDE) involving Riemann-Liouville differential operators of order $0<q<1$,

$$
\left.\begin{array}{c}
\frac{d}{d t}\left[\frac{x(t)}{f(t, x(t))}\right]=g(t, x(t)), t \in[0,1], \\
x(0)=x(1)=0
\end{array}\right\}
$$

where $f \in C([0, T] \times \mathbb{R}, \mathbb{R} \backslash\{0\})$ and $g \in C([0, T] \times \mathbb{R}, \mathbb{R})$, was studied in the works [2],[5].They established the existence and uniqueness results for fractional hybrid differential equations and initiated to study of such systems. 
In this paper we study the existence and extremal solution with the boundary value problem for the following nonlinear hybrid fractional differential equation (NHFDE)

$$
\begin{aligned}
& D_{0^{+}}^{\alpha}\left[\frac{x(t)}{f(t, x(t))}\right]+g(t, x(t))=0, \quad 0<t<1 \\
& x(0)=x(1)=x^{\prime}(0)=0,
\end{aligned}
$$

where $1<\alpha \leq 2$ is a real number and $D_{0^{+}}^{\alpha}$ is the standard Riemann Liouville fractional derivative. The result has been obtained an existence and extremal solutions for the boundary value problem for above equation(1.1) by using hybrid fixed point theorem, two operators in Banach Algebra due to Dhage.

Let $\mathbb{R}$ be the real line and $J=[0,1]$ be a bounded interval in $\mathbb{R}$. Let $C\left(J \times \mathbb{R}_{+}, \mathbb{R}\right)$ denote the class of continuous functions $\left(f: J \times \mathbb{R}_{+}, \mathbb{R}\right)$ and let $C\left(J \times \mathbb{R}_{+}, \mathbb{R}\right)$ denote the class of functions $g:\left(J \times \mathbb{R}_{+} \rightarrow \mathbb{R}\right)$ such that

i. $\quad$ the map $t \mapsto g(t, x)$ is measurable for each $x \in \mathbb{R}$, and

ii. the map $x \mapsto g(t, x)$ is continuous for each $t \in J$.

The class $C\left(J \times \mathbb{R}_{+}, \mathbb{R}\right)$ is called the Caratheodory class of functions on $J \times \mathbb{R}$ which are Lebesgue integrable when bounded by a Lebesgue integrable function on $J$.

In this paper, we assume $f \in C([0,1] \times \mathbb{R} \backslash\{0\})$ and $g \in C\left([0,1] \times \mathbb{R}_{+}, \mathbb{R}\right)$.

In section 2 we shall give some definitions and Lemmas to prove the main theorems. Section 3 establishes the existence theory. In section 4 , we establish the existence of solution of boundary value problem of equation (1.1) and (1.2) by using hybrid fixed point theorem in Banach algebras due to Dhage. Section 5 deals with solving the existence of extremal solutions. Finally we conclude by illustrating the main result by two examples in section 6 .

\section{Preliminaries}

We recall some basic definitions, lemmas and known results for fractional calculus and functional analysis to prove the main theorems, which can be found in the works; see [1], [2], [4], [7], and [8].

Definition 2.1 [12]. The Riemann-Lowville fractional derivative of order $\alpha>0$ of a function $f:(0,+\infty) \rightarrow \mathbb{R}$ is given by

$$
D_{0^{+}}^{\alpha} f(t)=\frac{1}{\Gamma(n-\alpha)} \frac{d^{n}}{d t} \int_{0}^{t} \frac{f(s)}{(t-s)^{\alpha-n+1}} d s,
$$

where $n=[\alpha]+1,[\alpha]$ denotes the integer part of number $\alpha$, provided that the right side is point wise defined on $(0,+\infty)$.

Definition 2.2[12]. The Riemann-Liouville fractional integral of order $\alpha>0$ of a function $f:(0,+\infty) \rightarrow \mathbb{R}$ is given by $\quad I_{0}^{\alpha} f(t)=\frac{1}{\Gamma(\alpha)} \int_{0}^{t}(t-s)^{\alpha-1} f(s) d s$,

provided that the right side is point wise defined on $(0,+\infty)$.

From the definition of the Riemann-Liouville derivative, we can obtain the following statements.

Definition 2.3 [2]. A mapping $\phi: \mathbb{R}_{+} \rightarrow \mathbb{R}_{+}$is called dominating function or in short $D$ - function if it is continuous and monotonic non-decreasing function satisfies $\emptyset(0)=(0)$.

Definition 2.4 [2]. Let $X$ be the Banach algebra with norm. A mapping $G: E \rightarrow E$ is called $D-$ Lipschitz or nonlinear $D$ - Lipschitz, if there exists a continuous non-decreasing function $\emptyset: \mathbb{R}_{+} \rightarrow$ $\mathbb{R}_{+}$such that 
$\|G x-G y\| \leq \emptyset\|x-y\|$, for all $x, y \in X$, where $\emptyset(0)=0$.

If $\emptyset(r)=k r, k>0$, then $k$ is called $G$-Lipschitz constant.

If $k<1, G$ is called contraction with contraction constant $k$.

Finally $G$ is called nonlinear $D-$ contraction if it is nonlinear $D$ - Lipschitz with $\emptyset(r)<r$ with $r>0$.

Lemma 2.1[18].Let $\alpha>0$. Assume that $u \in C(0,1) \cap L(0,1)$, then the fractional differential equation $D_{0^{+}}^{\alpha} u(t)=0$ has a unique solution

$$
u(t)=c_{1} t^{\alpha-1}+c_{2} t^{\alpha-2}+\cdots+c_{n} t^{\alpha-n}, c_{i} \in \mathbb{R}, i=1,2, \ldots, n ;
$$

where $n$ is the smallest integer greater than or equal to $\alpha$.

Lemma 2.2[18].Assume that $u \in C(0,1) \cap L(0,1)$ with a fractional derivative of order $\alpha>o$ that belongs to $C \in C(0,1) \cap L(0,1)$.Then

$$
I_{0^{+}}^{\alpha} D_{0^{+}}^{\alpha} u(t)=u(t)+c_{1} t^{\alpha-1}+c_{2} t^{\alpha-2}+\cdots+c_{n} t^{\alpha-n},
$$

for some $C_{i} \in \mathbb{R}$, where $i=1,2, \ldots, n, n=[\alpha]+1$, and $n$ is the smallest integer greater than or equal to $\alpha$. In the following, we present the Green function of fractional differential equation with boundary value problem.

Lemma 2.3.[18] Let $y \in C[0,1]$ and $1<\alpha \leq 2$. The unique solution of problem

$$
\begin{aligned}
& D_{0^{+}}^{\alpha}\left[\frac{x(t)}{f(t, x(t))}\right]+g(t, x(t))=0, \quad 0<t<1 \\
& x(0)=x(1)=0 \\
& \text { is } \quad x(t)=f(t, x(t)) \int_{0}^{1} G(t, s) y(s) d s \\
& \text { where } G(t, s)= \begin{cases}\frac{[t(1-s)]^{\alpha-1}-(t-s)^{\alpha-1}}{\Gamma(\alpha)}, & 0 \leq s \leq t \leq 1 \\
\frac{[t(1-s)]^{\alpha-1}}{\Gamma(\alpha)}, & 0 \leq t \leq s \leq 1\end{cases}
\end{aligned}
$$

Here $G(t, s)$ is called the Green function of boundary value problem (2.1) and (2.2).

We apply hybrid fixed point theorem due to Dhage [8] for proving the existence result.

Lemma 2.5[4].Let $S$ be a non-empty, closed convex and bounded subset of the Banach algebra $X$ and let $A: X \rightarrow X$ and $B: S \rightarrow X$ be two operators such that

(a) $A$ is Lipschitzian with a Lipschitz constant $\alpha$,

(b) $B$ is completely continuous,

(c) $x=A x B y \Rightarrow x \in S \forall y \in S$, and

(d) $\alpha M<1$, where $M=\|B(s)\|=\sup \{\|B(x)\|: x \in S\}$.

Then the operator equation $A x B x=x$ has a solution in $S$.

\section{Existence Theory}

In this section, we prove the existence result for fractional differential equation (1.1) on $J=[0,1]$ under the Lipschitz and Caratheodory conditions on nonlinearities involved in it. We place the fractional differential equation (1.1) in the space $B C\left(J, \mathbb{R}_{+}\right)$of continuous real-valued function defined on $J$. For convenience, we denote $T=\int_{0}^{1} G(s, s) d s$. 
Lemma 3.1[14]. The function $G(t, s)$ defined by (2.3) satisfies the following conditions:

1) $G(t, s)=G(1-s, 1-t)$, for $t, s \in(0,1)$;

2) $t^{\alpha-1}(1-t) s(1-s)^{\alpha-1} \leq \Gamma(\alpha), G(t, s) \leq(\alpha-1) s(1-s)^{\alpha-1}$, for $t, s \in(0,1)$;

3) $G(t, s)>0$, for $t, s \in(0,1)$; and

4) $t^{\alpha-1}(1-t) s(1-s)^{\alpha-1} \leq \Gamma(\alpha), G(t, s) \leq(\alpha-1) s(1-s)^{\alpha-1}$, for $t, s \in(0,1)$.

Let $X=B C\left(J, \mathbb{R}_{+}\right)$denotes the function space of all continuous real valued function on $J$. Define $\|$. and multiplication on $E$ by

$$
\begin{gathered}
\|x\|=\sup _{t \in J}|x(t)| \\
(x, y)(t)=x(t) y(t), \forall t \in J .
\end{gathered}
$$

Let $X=B C\left(R_{+}, \mathbb{R}\right)$ be the space of absolutely continuous function on $\mathbb{R}_{+}$and let $\Omega$ be the subset of $X$. Let a mapping $G: X \rightarrow X$ be an operator and consider the following equations in $X$ namely, $x(t)=$ $(G x)(t) \quad \forall t \in \mathbb{R}_{+}$. Clearly $X=B C\left(\mathbb{R}_{+}, \mathbb{R}\right)$ becomes a Banach space with respect to above norm and multiplication. By $L^{1}\left(\mathbb{R}_{+}\right)$we denote the space of Lebesguge integrable function defined on $J$ with the norm $\|\cdot\|_{L^{1}}$ defined by

$$
\|h\|_{L^{1}}=\int_{0}^{T}|G(s)| d s
$$

Denote $L^{1}(a, b)$ as the Lebesuge intergable function on interval $(a, b)$.Let $x \in L^{1}(a, b)$ and $\alpha>0$ be a fixed number.

Definition 3.1 [6]: A Mapping $g: \mathbb{R}_{+} \times \mathbb{R} \rightarrow \mathbb{R}$ is Caratheodory if;

i) $\quad t \rightarrow g(t, x)$ is measurable for each $x \in \mathbb{R}$ and

ii) $\quad t \rightarrow g(t, x)$ is continuous almost everywhere for $t \in \mathbb{R}_{+}$.

Furthermore Caratheodory function $g$ is $L^{1}$ - Caratheodory if

iii) For each real number $r>0$ there exist a function $h_{r} \in L^{1}\left(\mathbb{R}_{+}, \mathbb{R}\right)$ such that

$$
|g(t, x)| \leq h_{r}(t) \text { i.e. } t \in \mathbb{R}_{+} \forall x \in \mathbb{R} \text { with }|x| \leq r .
$$

Finally a Caratheodory function $g$ is $L_{X}^{1}$ - Caratheodory if;

iv) There exists a function $h \in L^{1}\left(\mathbb{R}_{+}, \mathbb{R}\right)$ such that

$$
|g(t, x)| \leq h_{r}(t) \text { i.e.t } \in \mathbb{R}_{+} \forall x \in \mathbb{R} .
$$

For convenience, the function $h$ is referred as a bound function for $g$.

\section{Main Results}

We need the following hypothesis for existence of solution of NHFDE.

$\left(\mathbf{H}_{1}\right)$ : the function $f: J \times \mathbb{R}_{+} \rightarrow \mathbb{R}$ is continuous and bounded with bound $\quad F=\sup _{(t, x) \in J}|f(t, x)|$;

$\left(\mathbf{H}_{2}\right)$ : there exists a constant $L>0$ such that $|f(t, x)-f(t, y)| \leq L|x-y|, \forall t \in J$ and $x, y \in \mathbb{R}$;

$\left(\mathbf{H}_{3}\right)$ there exists a function $h \in L^{1}\left(J, \mathbb{R}_{+}\right)$such that $|g(t, x)| \leq h(t), t \in J, \forall x \in \mathbb{R}_{+}$; and

$\left(\mathbf{H}_{4}\right)$ : the function $v: \mathbb{R}_{+} \rightarrow \mathbb{R}$ defined by the formula 


$$
v(t)=\int_{0}^{t} \frac{h(s)}{(t-s)^{1-\alpha}} d s=T\|h\|_{L^{1}} \quad \text { is bounded on } \mathbb{R}_{+} \text {and vanish at infinity, for all } x \in \mathbb{R}_{+} .
$$

Remark 4.1: If the hypothesis $\left(\mathrm{H}_{3}\right)$ and $\left(\mathrm{H}_{4}\right)$ hold, then there exists a constant $k_{1}>0$ such that

$$
k_{1}=\sup _{t \geq o}\left\{\frac{v(t)}{\Gamma(\alpha)}: t \in \mathbb{R}_{+}\right\}
$$

Theorem 4.1. Assume that the hypotheses $\left(\mathrm{H}_{1}\right)$ and $\left(\mathrm{H}_{4}\right)$ hold. Further, if

$$
\mathrm{LT}\|\mathrm{h}\|_{\mathrm{L}^{1}}<1,
$$

Then the boundary value problems (1.1) and (1.2) has a solution in the space $B C\left(\mathbb{R}_{+}, \mathbb{R}\right)$ and are locally attractive on $\mathbb{R}_{+}$.

Proof. Set $\mathrm{X}=\mathrm{BC}(J, \mathbb{R})$ and a subset $S$ of $X$ defined by

$$
\begin{aligned}
& s=\{x \in X \mid\|x\| \leq N\}, \\
& \text { where } N=\frac{f_{0} T\|h\|_{\mathrm{L}^{1}}}{1-L T\|h\|_{\mathrm{L}^{1}}} \text { and } f_{0}=\sup _{t \in J}|f(t, 0)| .
\end{aligned}
$$

Clearly $S$ is a closed, convex and bounded subset of the Banach space $X$. In view of Lemma (2.3), boundary value problem (1.1) and (1.2) is equivalent to the equation

$$
x(t)=f(t, x(t)) \int_{0}^{1} G(t, s) g(s, x(s)) d s, t \in J .
$$

Define two operators

$$
\begin{aligned}
& \quad A x(t)=f(t, x(t)), t \in J . \\
& \text { and } B x(t)=\int_{0}^{1} G(t, s) g(s, x(s)) d s, t \in J .
\end{aligned}
$$

Then equation (3.3) is transformed into the operator equation as

$$
A x(t) B x(t)=x(t), \quad t \in J .
$$

We shall show that the operators $A$ and $B$ satisfy all the conditions of Lemma 2.5.

Step I: We show that $A$ is Lipschitz on $X=B C\left(\mathbb{R}_{+}, \mathbb{R}\right)$.

Let $x, y \in X$, then the hypothesis $\mathbf{H}_{\mathbf{1}}$ is

$$
|A x(t)-A y(t)|=|f(t, x(t))-f(t, y(t))| \leq L\|x-y\|, \forall t \in J .
$$

Taking supremum over $t$, we obtain

$$
\|A x-A y\| \leq L\|x-y\|, \forall x, y \in X .
$$

Thus $A$ is Lipschitz on $X$ with Lipschitz constant $L$.

Step II: Now we show that $B$ is compact operator on $S$ into $X$. First we show that $B$ is continuous on $S$.

Let $\left\{x_{n}\right\}$ be a sequence in $S$ converging to a point $x \in S$. Then by Lebesgue dominated convergence theorem, for $t \in J$, we obtain

$$
\begin{aligned}
\lim _{n \rightarrow \infty} B x_{n}(t) & =\lim _{n \rightarrow \infty} \int_{0}^{1} G(t, s) g\left(s, x_{n}(s)\right) d s=\int_{0}^{1} G(t, s) \lim _{n \rightarrow \infty} g\left(s, x_{n}(s)\right) d s \\
& =\lim _{n \rightarrow \infty} \int_{0}^{1} G(t, s) g\left(s, x_{n}(s)\right) d s \\
& =B x(t), \forall t \in J .
\end{aligned}
$$


Taking supremum over $t$, shows that $B$ is continuous operator on $X$.

Sep III: Next we show that $B$ is a compact operator on $X$. For this, it is enough to show that $B(S)$ is uniformly bounded and equi-continuous set on $X$. Let $x \in S$ be an arbitrary. Then by hypothesis $\left(\mathrm{H}_{3}\right)$,

$$
\begin{aligned}
|B x| & =\left|\int_{0}^{1} G(t, s) g(s, x(s)) d s\right| \leq \int_{0}^{1} G(t, s)|g(s, x(s))| d s \leq \int_{0}^{1} G(s, s) h(s) d s \\
& \leq T\|h\|_{L^{1}}=v(t), \forall t \in J \text { and } \forall x \in S .
\end{aligned}
$$

This shows that $B$ is uniformly bounded on $S$.

On the other hand, let $t_{1}, t_{2} \in J$ with $t_{1}<t_{2}$, then for any $x \in S$, we have

$$
\begin{aligned}
& \left|B x\left(t_{2}\right)-B x\left(t_{1}\right)\right| \\
& \quad=\left|\int_{0}^{1} G\left(t_{2}, s\right) g(s, x(s)) d s-\int_{0}^{1} G\left(t_{2}, s\right) g(s, x(s)) d s\right| \\
& \quad \leq\|h\|_{L^{1}}\left|\int_{0}^{t_{2}} \frac{\left[t_{2}(1-s)\right]^{\alpha-1} d s-\left(t_{2}-s\right)^{\alpha-1}}{\Gamma(\alpha)}+\int_{t_{2}}^{1} \frac{\left[t_{2}(1-s)\right]^{\alpha-1}}{\Gamma(\alpha)} d s-\int_{0}^{t_{1}} \frac{\left[t_{1}(1-s)\right]^{\alpha-1}-\left(t_{1}-s\right)^{\alpha-1}}{\Gamma(\alpha)} d s-\int_{t_{1}}^{1} \frac{\left[t_{1}(1-s)\right]^{\alpha-1}}{\Gamma(\alpha)} d s\right| \\
& \quad \leq\|h\|_{L^{1}} \int_{0}^{1} \frac{\left[t_{2}^{\alpha-1}-t_{1}^{\alpha-1}\right](1-s)^{\alpha-1}}{\Gamma(\alpha)} d s+\|h\|_{L^{1}} \int_{0}^{t_{1}} \frac{\left(t_{2}-s\right)^{\alpha-1}-\left(t_{1}-s\right)^{\alpha-1}}{\Gamma(\alpha)} d s+\|h\|_{L^{1}} \int_{0}^{t_{1}\left(t_{2}-s\right)^{\alpha-1}} \frac{}{\Gamma(\alpha)} d s \\
& \quad \leq \frac{\|h\|_{L^{1}}\left[t_{2}^{\alpha-1}-t_{1}^{\alpha-1}+t_{2}^{\alpha}-t_{1}^{\alpha}\right]}{\Gamma(\alpha+1)} \\
& \quad \leq T\|h\|_{L^{1}}\left[\left|t_{2}^{\alpha}-t_{1}^{\alpha}-\left(t_{2}-t_{1}\right)^{\alpha}\right|+\left(t_{2}-t_{1}\right)^{\alpha}\right] \\
& \quad \leq\left[v\left(t_{2}\right)-v\left(t_{1}\right)\right] .
\end{aligned}
$$

Thus the right hand of above inequality does not depend on $X$ and tends to zero.

Therefore $\left|B x\left(t_{2}\right)-B x\left(t_{1}\right)\right| \rightarrow 0$ as $t_{1} \rightarrow t_{2}$. Hence for $t_{1}, t_{2} \geq T$ we have

$$
\begin{aligned}
\left|B x\left(t_{2}\right)-B x\left(t_{1}\right)\right| & =\left|t_{2} \int_{0}^{t_{2}} G\left(t_{2}, s\right) g\left(s, x_{n}(s)\right) d s-\int_{0}^{t_{1}} G\left(t_{2}, s\right) g\left(s, x_{n}(s)\right) d s\right| \\
& \leq\left|\int_{0}^{t_{2}} h(s) d s-\int_{0}^{1} h(s) d s\right| \leq\left|\int_{0}^{t_{2}} h(s) d s\right|+\left|\int_{0}^{1} h(s) d s\right| \\
& \leq v\left(t_{2}\right)+v\left(t_{1}\right) \\
& \leq \frac{\alpha}{2}+\frac{\alpha}{2} \leq \alpha \text { as } t_{1} \rightarrow t_{2} .
\end{aligned}
$$

Hence $\forall t_{1}, t_{2} \in \mathbb{R}_{+}$we have

$$
\left|B x_{n}\left(t_{2}\right)-B x_{n}\left(t_{1}\right)\right| \leq\left|B x_{n}\left(t_{2}\right)-B x_{n}(T)\right|+\left|B x_{n}(T)-B x_{n}\left(t_{1}\right)\right|
$$

If $t_{1} \rightarrow t_{2}, t_{1} \rightarrow T$ and $T \rightarrow t_{2}$ then

$$
\left|B x_{n}\left(t_{2}\right)-B x_{n}\left(t_{1}\right)\right| \rightarrow 0,\left|B x_{n}\left(t_{2}\right)-B x_{n}(T)\right| \rightarrow 0 \text { and }\left|B x_{n}(T)-B x_{n}\left(t_{1}\right)\right| \rightarrow 0 .
$$

Hence $\left\{B x_{n}\right\}$ is an equicontinuous sequence in $X=B C\left(\mathbb{R}_{+}, \mathbb{R}\right)$ and is relatively compact by the Arzela-Ascoli Theorem. As a result, $B$ is completely continuous operator on $S$.

Step IV: We show that the hypothesis (c) of Lemma 2.5 is satisfied.

Let $x \in X$ and $y \in Y$ be arbitrary such that $x=A x B y$ then by assumptions $\left(\mathrm{H}_{1}-\mathrm{H}_{3}\right)$, we have 


$$
\begin{aligned}
|x(t)|=|A x(t)||B y(t)| & =|f(t, x(t))|\left|\int_{0}^{1} G(t, s) g(s, y(s)) d s\right| \\
& \leq[|f(t, x(t))-f(t, 0)|+|f(t, 0)|]\left(\int_{0}^{1} G(s, s) g(s, y(s)) d s\right) \\
& \leq\left[L|x(t)|+F_{0}\right]|T|\|h\|_{L^{1}}
\end{aligned}
$$

Thus $\quad|x(t)| \leq \frac{F_{0} T\|h\|_{L^{1}}}{1-L T\|h\|_{L^{1}}}$

Taking supremum over, $t \in \mathbb{R}_{+}$, we obtain $\|x\| \leq \frac{F_{0} T\|h\|_{L^{1}}}{1-L T\|h\|_{L^{1}}}=N$.

This shows that the hypothesis of Lemma (2.5) is satisfied. Finally, we have

Step V: $M=\|B(S)\|=\sup \{\|B x\|: x \in S\}$

$$
\begin{aligned}
& \leq \sup \left\{\sup _{t \in J}\left|\int_{0}^{1} G(t, s) g(s, x(s)) d s\right|: x \in S\right\} \leq \sup \left\{\sup _{t \in J}\left|\int_{0}^{1} h(s) d s\right|: x \in S\right\} \\
& \leq \sup \left\{\sup _{t \in J}\left[T\|h\|_{L^{1}}\right]: x \in S\right\} \\
& \leq K_{1}
\end{aligned}
$$

Therefore $M K=L K_{1}<1$. Thus the hypothesis (d) of Lemma 2.5 is satisfied.

Hence all the conditions of Lemma 2.5 are satisfied and hence the operator equation $x=A x B y$ has a solution in $S$. As a result, the boundary value problem (1.1) and (1.2) has a solution defined on J. This completes the proof.

Remark 4.1: For the special case $f(t, x)=1$, we can find the corresponding existence result in Bai and $\mathrm{Lu},(\mathrm{see}[1])$.

Step VI: Finally we show the locally attractivity of solution for NHFDE (1.1 and 1.2).

Let $x$ and $y$ be two solutions of equation (1.1) and (1.2) in $X$ defined on $\mathbb{R}_{+}$. Then we have

$$
\begin{aligned}
|x(t)-y(t)| & =\mid[f(t, x(t))]\left[\left|\int_{0}^{1} G(t, s) g(s, x(s)) d s\right|\right]+[f(t, y(t))]\left[\left|\int_{0}^{1} G(t, s) g(s, y(s)) d s\right|||\right. \\
& \leq\left|[f(t, x(t))]\left[\int_{0}^{1} G(t, s) g(s, x(s))\right]\right| d s+\left|[f(t, y(t))]\left[\int_{0}^{1} G(t, s) g(s, y(s))\right]\right| d s \\
& \leq F\left\{\int_{0}^{1} G(s, s) h(s) d s\right\}+F\left\{\int_{0}^{1} G(s, s) h(s) d s\right\} \\
& \leq 2 F \int_{0}^{1} G(s, s) h(s) d s \\
& \leq 2 F T\|h\|_{L^{1}} \\
& \leq 2 F[v(t)] \quad\left[\because T\|h\|_{L^{1}}=v(t)\right]
\end{aligned}
$$

Since $\lim _{t \rightarrow \infty} v(t)=0$, so that $\lim _{t \rightarrow \infty}|x(t)-y(t)|=0$. This completes the proof.

\subsection{Existence of the Extremal Solution of NHFDE (1.1)}

A nonempty closed set $K$ in Banach Algebra $X$ is called a cone with vertex 0 if

i) $K+K \subseteq K$, ii) $\lambda K \subseteq K$ for $\lambda \in \mathbb{R}_{+}, \lambda \geq 0$, iii) $(-K) \cap K=0$, where 0 is the element of $X$.

A cone $K$ is said to be positive of $K \circ K \subseteq K$, where $\circ$ is a multiplication composition in $X$.

We introduce an order relation $\leq$ in $X$ as follows. 
Let $x, y \in X$. Then $x \leq y$ if and only if $y-x \in K$. A cone $K$ is said to be normal if the norm $\|$.$\| is$ semi-monotone increasing on $K$, that is there is a constant $N>0$ such that

$$
\|x\| \leq N\|y\|, \forall x, y \in K \text { with } x \leq y .
$$

It is known that if the cone $K$ is normal in $X$, then every order bounded set in $X$ is norm-bounded. These concepts appear in the works of Heikkila and Lakshmikantham (see, [3], [10]).

Lemma 5.1 [6]: Let $K$ be a positive cone in a real Banach Algebra $X$ and let $u_{1}, u_{2}, v_{1}, v_{2} \in K$ be such that

$$
u_{1} \leq v_{1}, u_{2} \leq v_{2} \text { then } u_{1} u_{2} \leq v_{1} v_{2} \text {. }
$$

We use the following fixed point theorem due to Dhage [5] for proving the existence of extremal solution for BVP (2.1) under certain monotonically conditions.

Lemma 5.2[6]: Let $K$ be a cone in Banach Algebra $X$ and let $a, b \in X$ be such that $a \leq b$. Suppose that $A, B:[a, b] \rightarrow K$ are two nondecrasing operators such that

a) $A$ is Lipschitzian with a Lipschitz constant $\alpha$.

b) $B$ is complete,

c) $A x B x \in[a, b] \forall x \in[a, b]$.

Further, if the cone $K$ is positive and normal, then the operator equation $A x B x=x$ has a least and a greatest positive solution in $[a, b]$ whenever $\alpha M<1$, where $M=\|B(s)\|=\sup \{\|B(x)\|: x \in[a, b]\}$.

We equip the space $B C\left(J, \mathbb{R}_{+}\right)$with order relation $\leq$with the help of the cone $K$ defined by

$$
K=\{x \in B C(J, \mathbb{R}): x(t) \geq 0 \forall t \in J .
$$

It is well known that cone $K$ is positive and normal in $B C\left(J, \mathbb{R}_{+}\right)$.

Definition 5.2 ([2], [16]): A function $u \in B C\left(\mathbb{R}_{+}, \mathbb{R}\right)$ is called a lower function of NHFDE on $\mathbb{R}_{+}$if the function $t \rightarrow \frac{u(t)}{f(t, u(t))}$ is continuous and

$$
\begin{aligned}
& D_{0^{+}}^{\alpha}\left[\frac{u(t)}{f(t, u(t))}\right]+g(t, u(t))=0, \quad 0<t<1 \\
& x(0)=x(1)=0 .
\end{aligned}
$$

Again a function $v \in B C\left(\mathbb{R}_{+}, \mathbb{R}\right)$ is called a upper function of NHFDE on $\mathbb{R}_{+}$, if the function $t \rightarrow \frac{v(t)}{f(t, v(t))}$ is continuous and a function $a \in B C(J, \mathbb{R})$ is a solution of NHFDE is lower as well as an upper solution of the NHFDE defined on J.

We consider the following assumptions

(B) $f: J \times \mathbb{R} \rightarrow \mathbb{R}_{+}, g: J \times \mathbb{R} \rightarrow \mathbb{R}_{+}$.

$\left(\mathbf{B}_{2}\right)$ The NHFDE (2.1) has lower solution $a$ is an upper solution $b$ defined on $J$ with $a<b$.

(B) The function $x \rightarrow \frac{x}{f(t, x)}$ is increasing in the interval $\left[\min _{t \in J} a(t), \max _{t \in J} b(t)\right]$ almost on $J$ with $a<b$.

$\left(\mathbf{B}_{4}\right)$ The function $f(t, x)$ and $g(t, x)$ are nondecreasing in $x$ almost everywhere for $t \in J$.

$\left(\mathbf{B}_{5}\right)$ There exists a function $K \in L^{1}(J, \mathbb{R})$ such that $g(t, b(t)) \leq K(t)$.

In particular, the hypothesis $\left(\mathrm{B}_{5}\right)$ holds if $f$ is continuous and $g$ is $L^{1}-$ Caratheodory on $J \times \mathbb{R}$.

Theorem 5.1 Suppose that the assumptions $\left(\mathrm{H}_{2}\right)$ and $\left(\mathrm{B}_{1}-\mathrm{B}_{5}\right)$ hold. Further, if $T\|h\|_{L^{1}}<1$ (5.2) then NHFDE (2.1) has minimal and maximal positive solution defined on $J$.

\section{Proof:}

Now NHFDE (2.1) is equivalent to the integral equation (2.5) defined on $J$.

Let $X=B C(J, \mathbb{R})$. Define the two operators $A$ and $B$ on $X$ by the equations (4.5) and (4.6). Then the integral equation is transformed into an operator equation $A x(t) B x(t)=x(t)$ in Banach Algebra $X$. 
The hypothesis $\left(\mathrm{B}_{1}\right)$ implies $A, B:[a, b] \rightarrow K$. Since the cone $K$ in $X$ is normal, $[a, b]$ is normalbounded set in $X$. By the proof of Theorem 4.1, $A$ is Lipschitzian with Lipschtiz constant $L$ and $B$ is completely continuous operator on $[a, b]$. Again hypothesis $\left(\mathrm{B}_{4}\right)$ implies that $A$ and $B$ are nondecreasing on $[a, b]$. To see this, let $x, y \in[a, b]$ such that $x \leq y$. Then by hypothesis $\left(\mathrm{B}_{3}\right)$, we have

$$
A x(t)=f(t, x(t)) \leq f(t, y(t))=A y(t) \forall t \in J .
$$

Similarly, we have

$$
B x(t)=\int_{0}^{1} G(t, s) g(s, x(s)) d s \leq \int_{0}^{1} G(t, s) g(s, y(s)) d s=B y(t) \forall t \in J .
$$

So $A$ and $B$ are non-decreasing operators on $[a, b]$. By Lemma 5.1 and hypothesis (B4) together imply that

$$
\begin{aligned}
a(t) & \leq f(t, a(t)) \int_{0}^{1} G(t, s) g(s, a(s)) d s \leq f\left(t, x(t) \int_{0}^{1} G(t, s) g(s, x(s)) d s\right. \\
& \leq f\left(t, b(t) \int_{0}^{1} G(t, s) g(s, b(s)) d s\right. \\
& \leq b(t), \forall t \in \mathbb{R}_{+} \text {and } x \in[a, b] .
\end{aligned}
$$

So $a(t) \leq A x(t) B x(t) \leq b(t) \forall t \in \mathbb{R}_{+}$and $x \in[a, b]$. Hence $A x B x \in[a, b] \forall x \in[a, b]$

Again $M=\|B([a, b])\|=\sup \{\|B x\|: x \in[a, b]\}$

$$
\begin{aligned}
& \quad \leq \sup \left\{\sup _{t \in J} \int_{0}^{1}|g(s, x(s)) d s|: x \in[a, b]\right\} \\
& \leq T\|h\|_{L^{1}} \\
& \text { and so } \alpha M \leq T\|h\|_{L^{1}}<1 .
\end{aligned}
$$

Now we apply Lemma 5.2 for the operator $A x B x=x$ to yield NHFDE (2.1) to show minimal and maximal positive solution in $\mathbb{R}_{+}$. This completes the proof.

\subsection{Examples}

Example 5.1: Consider the boundary value problem

$$
\begin{aligned}
& D_{0^{+}}^{\frac{3}{2}} x(t)+\sin x=0,0<t<1, \\
& x(0)=x(1)=0
\end{aligned}
$$

Let $f(t, x(t))=1, g(t, x)=\sin x, h(t)=1$. Then for $\alpha=\frac{3}{2}$, hypothesis $\left(\mathrm{H}_{2}\right)$ and $\left(\mathrm{H}_{3}\right)$ hold, since

$$
T=\int_{0}^{1} G(s, s) d s=\int_{0}^{1} \frac{[s(1-s)]^{\alpha-1}}{\Gamma(\alpha)} d s=\int_{0}^{1} \frac{[s(1-s)]^{\frac{1}{2}}}{\Gamma\left(\frac{3}{2}\right)} d s=\frac{\sqrt{\pi}}{4} .
$$

Choosing $\mathrm{L}=1$ then $T\|h\|_{L^{1}}=\frac{\sqrt{\pi}}{4}<1$.Therefore boundary value problem (5.3) and (5.4) has a solution.

Example 5.2: Consider the boundary value problem for $\alpha=\frac{3}{2}$.

$$
\begin{aligned}
& D_{0^{+}}^{\alpha}\left[\frac{x(t)}{\sin x+2}\right]+\cos x=0, \quad 0<t<1 \\
& x(0)=x(1)=0
\end{aligned}
$$

Let $f(t, x)=\sin x+2, g(t, x)=\cos x, h(t)=1$. Then the hypothesis $\left(\mathrm{H}_{2}\right)$ and $\left(\mathrm{H}_{3}\right)$ hold.

Since $T=\frac{\sqrt{\pi}}{4}$, choosing $L=1$ then $T\|h\|_{L^{1}}=\frac{\sqrt{\pi}}{4}<1$.

Therefore the boundary value problem (5.5) and (5.6) has a solution.

\section{Conclusion}

In this work we have studied the existence solution for boundary value problem for nonlinear hybrid fractional differential equation. This topic may be applicable in numerous fields of science and engineering and currently it is a central point to the researchers. 
B.D. Karande E Pravin M. More/ Existence and Extremal Solution of Boundary Value Problem for ...

\section{Acknowledgements}

The authors are thankful to the referees for their constructive suggestions for the improvement of the content of the paper.

\section{References}

[1] Bai Z. \& Lu H. (2005). Positive solutions for boundary value problem of nonlinear fractional differential equation, Journal of Mathematical Analysis and Applications, 311(15):495-505.

[2] Banas J. and Dhage B.C. (2008). Globally asymptotic stability of solutions of functional integral equations, Non-linear functional analysis, 69 (7): 1945-1952.

[3] Dhage B.C. (1999). Fixed point theorem in ordered Banach algebras and applications, Panamerican, Mathematical Journal, 9: 83-102.

[4] Dhage B.C. (2004). A nonlinear alternative in Banach algebra with application to functional differential equations, Nonlinear Functional Analysis Applications. 8: 563-575.

[5] Dhage B.C. (2005). On fixed point theorem in Banach algebra with application, Applied Mathematics Letter, 18:273-80.

[6] Dhage B.C. and Karande B.D. (2005). First order integro-differential equations in Banach algebras involving Caratheodory and discontinuous nonlinearities; Electronic Journal of Qualitative Theory of Differential Equations, 21: 1-16.

[7] Dhage, B.C. and Lakshmikantham V. (2004). Basic result on hybrid differential equations, Nonlinear Analysis Math. Comput., 15(4): 621-640.

[8] Dhage B.C and Lakshmikantham V. (2010). Basic result on hybrid differential equations, Nonlinear Analysis Hybrid System, 4: 414-424.

[9] Geo D.J. and Lakshmikantham V.(1988). Nonlinear Problems in Abstract Cones, Academic Press Elsevier.

[10] Heikkilla S. and Lakshmikantham V. (1994). Monotone Iterative Technique for the Discontinuous Nonlinear Differential Equation, Marcel Deeker, New York.

[11] Karande B.D. (2013). Fractional order functional intgro-differential equation in Banach Algebras, Malaysian journal of mathematical sciences, 8: 1-16.

[12] Karande B. D. and Yachwad S. S. (2015). Existence of the locally attractive solution for a fractional order differential equations in Banach Algebra, International Journal of Research in Advent Technology , 3:634-642.

[13] Li C.F., Lou X.N. and Zhou Y. (2010). Existence of positive solution of boundary value problem for fractional differential equations, Computers and Mathematics wih Applications. 59 (3) : 1363-1375.

[14] Miller K.S. and Ross B. (1993). An Introduction to the Fractional Differential Equation, John Wiley, New York.

[15] Oldham K.B. and Spinier J. (1974). The Fractional Calculus, Academic Press, New York,

[16] Samko S.G., Kilbas A.A. and Marichev O.I. (1993). Fractional integral and derivative :theory and application, Gordon and Breach Science Publishers, USA.

[17] Sun S., Zhao Y., Han Z. and Li Y. (2012). The existence of solution for boundary value problem of fractional hybrid differential equation, Communication in Nonlinear Science and Numerical Simulation, Elsever, 17(12) : 4961-4967.

[18] Zhao Y. and Wang Y. (2014). Existence to solution to boundary value problem of a class of nonlinear fractional differential equations, Journal Advance in Differential equations, Springer, 1:174- 184. 